\title{
Evaluation of Diffusion Creep in Low Melting Point Materials by Nanoindentation Creep Test*
}

\author{
Tadahiro SHIBUTANI**, Qiang YU ${ }^{* *}$ and Masaki SHIRATORI**
}

\begin{abstract}
In this study, diffusion creep in $\mathrm{Sn}-37 \mathrm{~Pb}$ as a low melting point alloy during a nanoindentation creep test was examined. The creep exponent, $n$, from the relationship between hardness and indenter dwell time decreases as a function of time and is saturated when $n=1$. From observations of the indented surface, plastic deformation due to the indenter takes place in the early stages. On the other hand, granular deformation takes place in the middle and last stages. Finite element analysis revealed that the reduction in Mises stress is faster than that of hydrostatic stress. Multiaxial stresses appear below the indenter, and axial components of stress remain after relaxation. Since the core hydrostatic stress causes a gradient in the chemical potential at grain boundaries, diffusion creep affects the behavior of indentation creep in the last stage. A transition from power-law creep to diffusion creep occurs below the indenter.
\end{abstract}

Key Words: Creep, Hardness, Finite Element Method, Nanoindentation, Diffusion Creep, and Low Temperature Melting Point Alloy

\section{Introduction}

At elevated temperatures, the mechanism of creep deformation in metals is classified into dislocation creep and diffusion creep ${ }^{(1)}$. Generally, dislocation creep (powerlaw creep) is dominant at higher stresses and elevated temperatures. Because diffusivity along or through grains is activated at higher temperatures, diffusion creep is dominant under lower stress and higher temperatures. The value of stress sensitivity during power-law creep is over 3 according to experimental results ${ }^{(2)}$. On the other hand, diffusion creep is characterized by a value of stress sensitivity of $n=1^{(3),(4)}$. According to the deformation map ${ }^{(5)}$, when diffusion creep is dominant, stress is extremely low under uniaxial stress. Since the test period for diffusion creep is long, few experimental reports have appeared about diffusion creep ${ }^{(5),(6)}$.

Several creep tests have been proposed: a tensile creep test, a torsional creep test, and a creep test of internally pressurized cylinders. In particular, the indentation creep test is used to measure creep in a localized

* Received 17th April, 2006 (No. 04-1330). Japanese Original: Trans. Jpn. Soc. Mech. Eng., Vol.71, No.710, A(2005), pp.1285-1291 (Received 20th December, 2004)

** Faculty of Engineering, Yokohama National University, 79-5 Tokiwadai, Hodogaya-ku, Yokohama 240-8501 Japan. E-mail: shibu@swan.me.ynu.ac.jp $\operatorname{area}^{(7)-(12)}$. Displacements into the surface are measured continuously under a constant load, and the creep properties are extracted from the evolution of the hardness on the basis of the power-law creep relationship. Indentation creep tests are widely employed as an approximate method, and the creep properties obtained from indentation tests correspond with that from other creep tests. However, because not only stress but also strain rate varies during the test, the mechanism of creep deformation is complicated.

In this study, the mechanism of creep deformation in a localized area was examined using the nanoindentation creep test. The nanoindentation creep test was carried out on a low temperature melting point alloy, $\mathrm{Sn}-37 \mathrm{~Pb}$, in which creep takes place at room temperature. Triaxial stresses appeared below the indenter due to the surroundings. Then, hydrostatic stress after relaxation affected the diffusion creep. The focus of this study was the effect of diffusion creep in a localized area. Finite element analysis was used to identify the stress field near the indenter and the evolution of stress during the nanoindentation creep test.

\section{Theory of Creep Deformation}

Dislocation creep is found experimentally to obey a constitutive relation of the power-law form,

$$
\dot{\varepsilon}=A \sigma^{n}
$$


in the uniaxial tensile state, where $A$ and $n$ are the creep constant and exponent, respectively. Generally, the value of the creep exponent, $n$, ranges from 3 to 8 in metals. This equation is generalized for states of arbitrary stress and strain rates using the Mises equivalent stress and strain rates. With the assumption to no effect of hydrostatic pressure on strain rate, the components of stress and strain rates can be expressed as follows: ${ }^{(13)}$

$$
\dot{\varepsilon}_{i j}=C \bar{\sigma}^{n-1} s_{i j} .
$$

Here, $s_{i j}=\sigma_{i j}-\delta_{i j} \sigma_{k k} / 3$ indicates deviatoric stress with the summation convention.

At elevated temperatures and relatively low stress, materials often deform because of the transportation of atoms, and diffusion creep takes place. Atoms move along grain boundaries or through grains. When grain boundary diffusion dominates $\left(0.5 T_{m}<T<0.8 T_{m}, T_{m}\right.$ : melting point), the creep is known as Coble creep; when, instead, bulk diffusion dominates $\left(0.8 T_{m}<T\right)$, it is often called Nabarro-Herring creep. The flux of atoms is driven by the chemical potential gradient of the atoms in the grain boundary according to the relationships ${ }^{(13),(14)}$

$$
J_{B}=-\frac{D_{B}}{k T} \frac{\partial \mu}{\partial s} \quad \text { along grain boundaries }
$$

and

$$
J_{V}=-\frac{D_{V}}{k T} \operatorname{grad} \mu \quad \text { in grains. }
$$

Here, $D_{B}$ and $D_{V}$ are the grain boundary and body diffusion coefficients, respectively, $k$ is Boltzmann's constant, and $T$ is the absolute temperature. The chemical potential, $\mu$, at the grain boundary is a function of the normal stress acting on the grain boundary, $\sigma_{n}$, and can be expressed as follows: $:^{(4),(15)}$

$$
\mu=\mu_{0}-\sigma_{n} \Omega,
$$

where $\mu_{0}$ is the potential of the bulk, and $\Omega$ is the atomic volume. Therefore, the deformation rate due to diffusion creep depends on the gradient of the stresses acting on grain boundaries. When hydrostatic stresses are applied, no diffusion creep takes place because no gradient of potential appears ${ }^{(16),(17)}$. However, when a load is applied at a localized area, the gradient of the hydrostatic stresses becomes the driving force for diffusion in materials. The relationship between stress, $\sigma_{0}$, and strain rate in the uniaxial stress state can be expressed as follows: $:^{(1)}$

$$
\begin{aligned}
\dot{\varepsilon}_{2} & =\dot{\varepsilon}_{B}+\dot{\varepsilon}_{V} \\
& =A_{0} \frac{\sigma_{0} \Omega}{k T} \frac{1}{d^{2}} D_{V}\left\{1+\frac{\pi \delta_{B}}{d} \frac{D_{B}}{D_{V}}\right\},
\end{aligned}
$$

where $d$ is the diameter of the grains, $\delta_{B}$ is the thickness of the diffusion layer, and $A_{0}$ is a constant. Therefore, this equation is equivalent to Eq. (1) with $n=1$ in a uniaxial stress state. The creep exponent often characterizes the mechanism of the creep deformation. In the case of a multiaxial stress state, the dependence on stress sensitivity was confirmed analytically ${ }^{(12),(18)}$.

\section{Experimental Procedure}

\subsection{Nanoindentation creep test}

The decrease in hardness as a function of the dwell time during indentation creep with a self-similar indenter has the following relationship, determined experimentally $^{(8)}$,

$$
-n \ln H=\ln B-\frac{Q}{R T}+\ln t,
$$

where the hardness, $H$, is defined as the maximum load, $P$, divided by the residual indentation, $S\left(=D_{1} h_{c}^{2}, D_{1}\right.$ : the shape parameter, $h_{c}$ : displacement into the surface). Because the hardness decreases as a function of the displacement due to creep, the $t-H$ curve remains linear on logarithmic scales. The slope of the $t-H$ curve ( $n$ in Eq. (7)) corresponds to the creep exponent in the tensile creep test. Thus, the indentation creep test is used to simplify the creep test $^{(9),(11)}$.

The stress state during indentation is different from that in the tensile creep test. Not only indented pressure but also stress due to the surroundings are generated, and a multiaxial stress state appears below the indenter. Therefore, the indentation creep test measures the creep properties under multiaxial compressive stresses. At the start of the dwell time, equivalent stress reaches the yield stress, and grain sliding or dislocation creep is dominant. The hardness decreases because of the increased displacement into the surface. Although the equivalent stress decreases as the dwell time increases, the axial stresses remain after relaxation due to the constraints of the indenter and the surroundings. The strain rate of power-law creep in Eq. (2) decreases rapidly. On the other hand, the chemical potential due to the normal stress at grain boundaries in Eq. (5) remains relatively high compared to that in the surrounding area. The diffusion of atoms takes place due to the gradient of potential from the indented area to the surroundings. Because the creep exponent of the diffusion creep is the lowest, diffusion creep is dominant after a long dwell time. Therefore, the transition from dislocation creep to diffusion creep can be determined during the nanoindentation creep test. The localized stress gradient activates atom transport near the indenter.

\section{2 Test method}

The tested material is a solder ball of $\mathrm{Sn}-37 \mathrm{~Pb}$ as a typical low melting point alloy (melting point: $456 \mathrm{~K}$ ). The diameter of the ball is $1.5 \mathrm{~mm}$, which is larger than the indent area. The microstructure of $\mathrm{Sn}-37 \mathrm{~Pb}$ is shown in Fig. 1. It consists of $\alpha$ - and $\beta$-phases. The white and black areas indicate $\beta$-Sn and $\alpha-\mathrm{Pb}$, respectively. The granular structure is generated due to recrystallization or quenching; the size of grain is enlarged to stabilize the microstructure. Because the diameter of the grains in used balls is about $15 \mu \mathrm{m}$, the mechanical properties can be evaluated without changing the structure ${ }^{(11)}$. The surface 


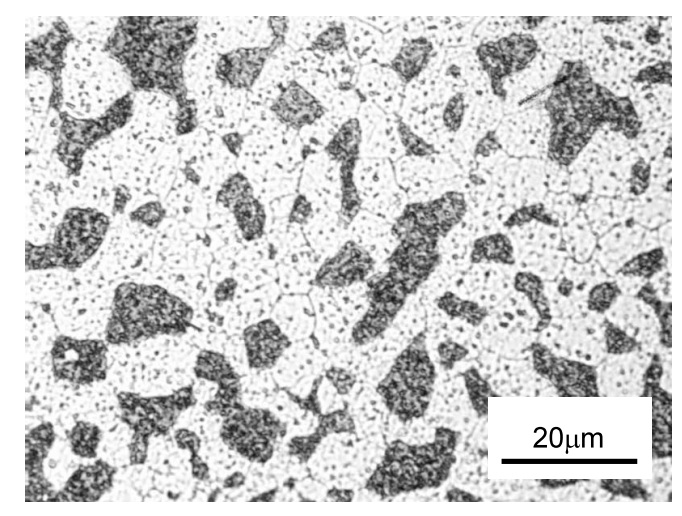

Fig. 1 Microstructure of $\mathrm{Sn}-37 \mathrm{~Pb}$

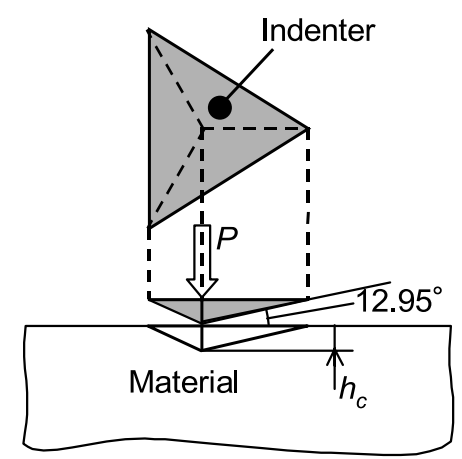

Fig. 2 Schematic illustration of nanoindentation

of the samples molded by resin is polished mechanically.

The nanoindentation test was performed using a MTS NanoindenterXP ${ }^{\mathrm{TM}}$. Figure 2 shows a schematic illustration of nanoindentation. This testing system used a Berkovich indenter, the curvature of which is below $50 \mathrm{~nm}$, and experiments were carried out at room temperature and in air. The applied maximum loads were 0.98, 9.8 , and $98 \mathrm{mN}$. After an indentation for $15 \mathrm{~s}$, displacements and applied load were measured continuously during the holding period. The resolutions of the displacement and load were $0.01 \mathrm{~nm}$ and $1 \mathrm{nN}$, respectively. The dwell time ranged from $3000 \mathrm{~s}$ to $72000 \mathrm{~s}$. Two experiments under each set of conditions were performed to assess repeatability.

\section{Finite Element Analysis}

To investigate the stress field below the indenter during nanoindentation creep, finite element analysis (FEA) was carried out. Figure 3 shows a schematic illustration of the analytical model. An axisymmetrical model consisting of an indenter and a solder ball was used to simplify the analysis. A Berkovich indenter was used in the nanoindentation test, but a conical indenter, the surface area of which was the same as the Berkovich indenter, was modeled in this analysis. Simulations were carried out using the commercial nonlinear structural solver, ANSYS. There were 3365 axisymmetrical elements and 3409 nodes in this model. Fine mesh was constructed near

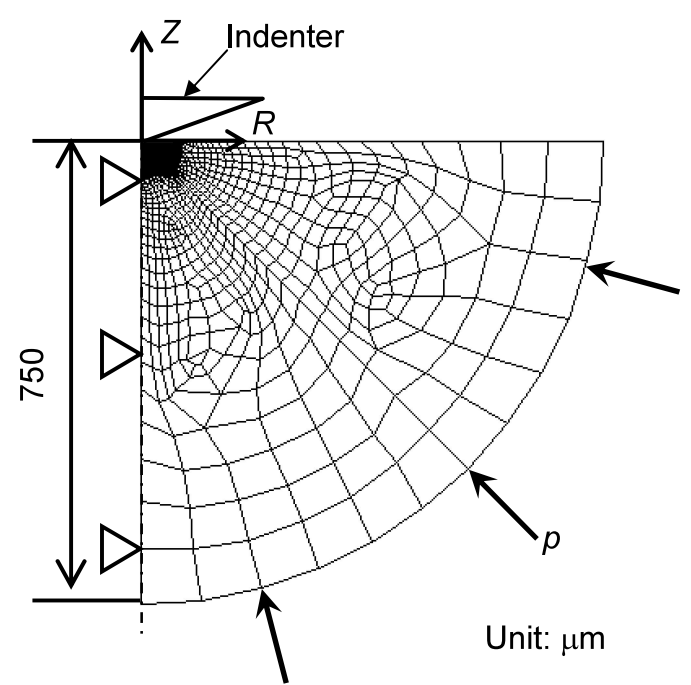

Fig. 3 Mesh division and boundary conditions for elasticplastic-creep

Table 1 Material properties for the eutectic of $\mathrm{Sn}-37 \mathrm{~Pb}^{(19)}$

\begin{tabular}{lc}
\hline Material property & Value \\
\hline Young's modulus $E$ & $20 \mathrm{GPa}$ \\
Poisson's ratio $u$ & 0.4 \\
Yield stress $\sigma_{Y}$ & $29.1 \mathrm{MPa}$ \\
Creep constant $A$ & $5.486 \times 10^{-12} \mathrm{MPa}^{-n} \cdot \mathrm{s}^{-1}$ \\
Creep exponent $n$ & 4.7 \\
\hline
\end{tabular}

the contact area and the minimum element size was set at $1 \mu \mathrm{m}$. Because the solder ball is soft, the indenter was assumed to be a rigid body. Displacements in the $x$ direction on the axisymmetrical axis were fixed. Pressure, $p=P / 2 \pi R^{2}(P=98 \mathrm{mN})$, was applied on the bottom of the solder as shown in Fig. 3. It has been reported that the effect of friction on deformation in an indentation is small. In this analysis, there was assumed to be no friction between the indenter and the surface of the material. Sn$37 \mathrm{~Pb}$ was assumed to be an elastic-plastic-creep body, and its material properties were assigned based on the tensile and creep tests reported in Ref. (19). For the quantitative evaluation of the nanoindentation, the material properties determined directly from tested materials should be used. However, in this study, the focus is the relaxation behavior during the nanoindentation creep test on the basis of power-law creep. Additionally, there are few differences between the material properties of bulk material and a solder ball. The constitutive equation for creep is the powerlaw in Eq. (1), and the holding time is set at $t=3000 \mathrm{~s}$. Because the loading time is much smaller than the dwell time, no effect of creep was considered. Table 1 summa- 
rizes the values of the material properties in this analy$\operatorname{sis}^{(19)}$.

\section{Results and Discussion}

\section{$5.1 t-H$ relationship}

Figure 4 shows the relationship between a load on a surface and displacement into a surface at $P=0.98,9.8$, and $98 \mathrm{mN}$. Both axes are logarithmic scales. Displacements increase as a function of dwell time, which implies that creep deformation takes place. The maximum displacement is $780 \mu \mathrm{m}$ in $P=0.98 \mathrm{mN}$, and the contact area expands to almost a grain size. On the other hand, displacements reaches $8-30 \mu \mathrm{m}$ in $P=9.8,98 \mathrm{mN}$, and the contact area consists of several grains. When $P<0.5 \mathrm{mN}$, the microstructure of $\mathrm{Sn}-37 \mathrm{~Pb}$ and the surface affects the behavior of the deformation on the surface. These curves correspond with one another for $P>0.5 \mathrm{mN}$. Figure 5 is the evolution of hardness plotted on logarithmic scales. Hardness, the maximum load divided by the contact area, decreases as creep proceeds. In the early stages, the curves remain linear in this graph and obey Eq. (7). The value of the slope corresponding to the creep exponent is $n=6$. It is

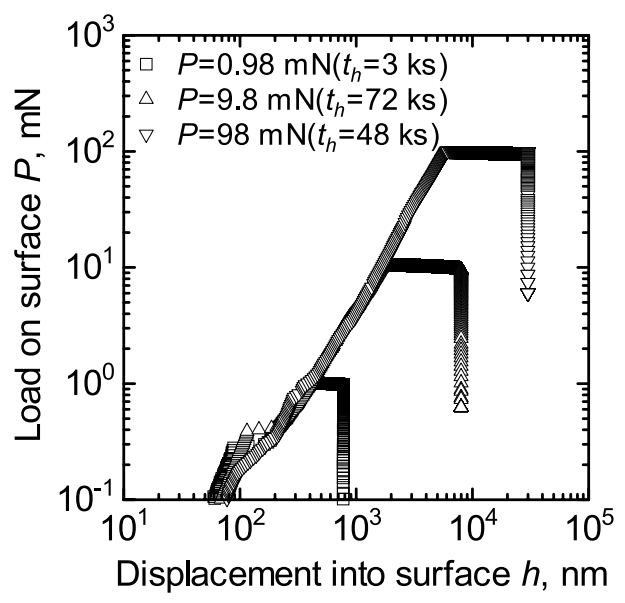

Fig. 4 Relationship between load on surface and displacement into surface

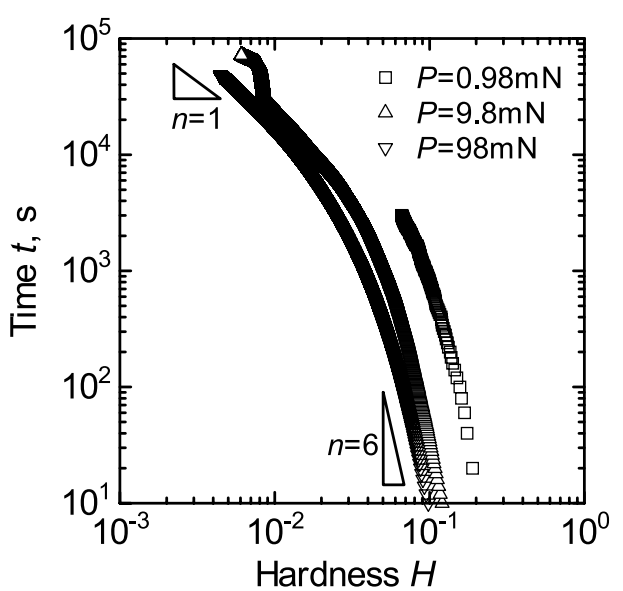

Fig. 5 Evolution of hardness in nanoindentation creep test larger in the other tensile or creep tests, but it agrees with the results from the nanoindentation creep test for a single crystal of tin. Therefore, the power-law creep is dominant in the early stages of indentation creep. After $1000 \mathrm{~s}$, the slope of the curve decreases for $P=9.8$ and $98 \mathrm{mN}$. This implies that the mechanism of the creep changes. The slope is saturated at $n=1$. In the case of $P=9.8 \mathrm{mN}$, the decrease in hardness is prevented during $t=30-60 \mathrm{ks}$. The value of the slope is over $n=6$, and the power-law creep does not take place. Either the grain boundary sliding is prevented, or the elasticity recovers due to the decrease in stress. The arrested hardness decreases to $n=1$ again after $t=60 \mathrm{ks}$. This implies that a transition from powerlaw creep to diffusion creep occurs. Because the melting point of $\mathrm{Sn}-37 \mathrm{~Pb}$ is relatively low $\left(T_{m}=456 \mathrm{~K}\right)$, the normalized temperature at room temperature $\left(T_{m}=300 \mathrm{~K}\right)$ is about 0.66 , at which the grain boundary diffusion creep is dominant at relatively low stress.

Figure 6 shows the morphology of the residual indented area and its cross-sectional profile at $P=9.8 \mathrm{mN}$. In this graph, the profile at $P=78.4 \mathrm{mN}$ and $t=30 \mathrm{~s}$ is also shown by the dashed line is almost the same shape as the indenter. The profile of the dashed line is shifted to the tip of the indenter in the case of $P=9.8 \mathrm{mN}$. For the line $\mathrm{a}-\mathrm{b}$, the solid line is in agreement with the dashed line. The plastic deformation due to the contact of the indenter takes place. On the other hand, the residual area during the holding time (line b-c) sinks into the material. This implies that another deformation mechanism arises during the holding period. The tilts of the grain surfaces in the residual contact area are described (line $c-d$ and $b^{\prime}-c^{\prime}$ ).

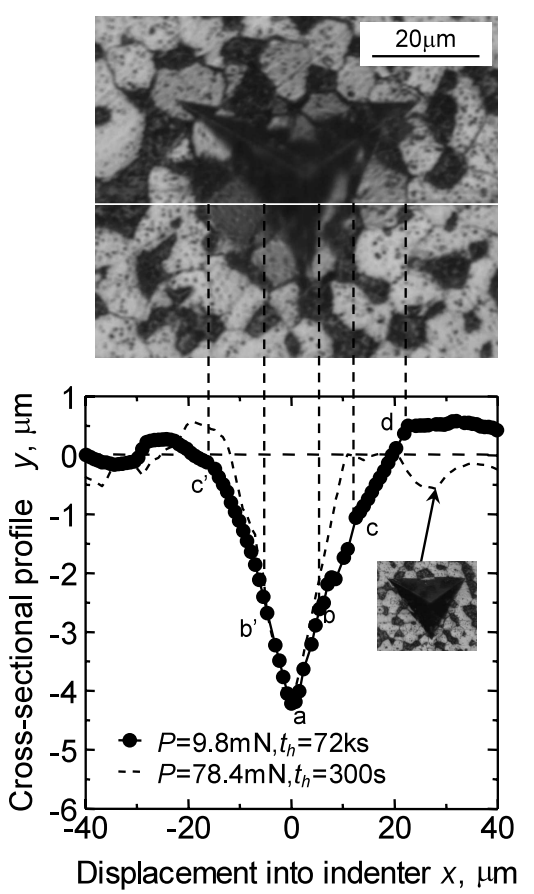

Fig. 6 Indentation profile and deformation of grains 
In polycrystalline materials, several grains are rotated due to grain boundary diffusion ${ }^{(16)-(18)}$. In the case of indentation creep, the rotated surface suggests that the grains have been rotated due to grain boundary diffusion or bulk diffusion. The effective zone expands into the adjacent grains with a residual contact area at $P=9.8 \mathrm{mN}$. At $P=98 \mathrm{mN}$, several grains are tilted.

\subsection{Relaxation due to dislocation creep}

Figure 7 (a) and (b) shows contour maps of equivalent stress and hydrostatic stress at $t=0 \mathrm{~s}$, respectively. Stress appears concentrated near the contact area, and the size of the plastic zone expands to $30 \mu \mathrm{m}$. The hydrostatic stress is higher than the equivalent stress. Because the equivalent stress decreases due to relaxation, triaxial stresses are residual. The maximum compressive principal stress is generated in the direction normal to the contact surface.

Figure 8 (a) shows the evolution of equivalent stress. The horizontal axis indicates the distance into the surface. The values plotted are taken at adjacent integration points with respect to the axissymmetrical axis $(x=0)$ and are normalized by the yield stress. The equivalent stress decreases for $h_{r}<40 \mu \mathrm{m}$. According to Eq. (1), a $40 \%$ decrease in equivalent stress causes a $99 \%$ decrease in strain

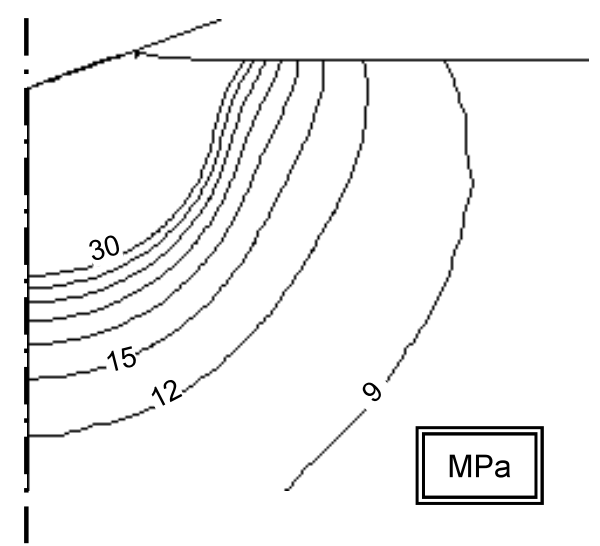

(a) Equivalent stress

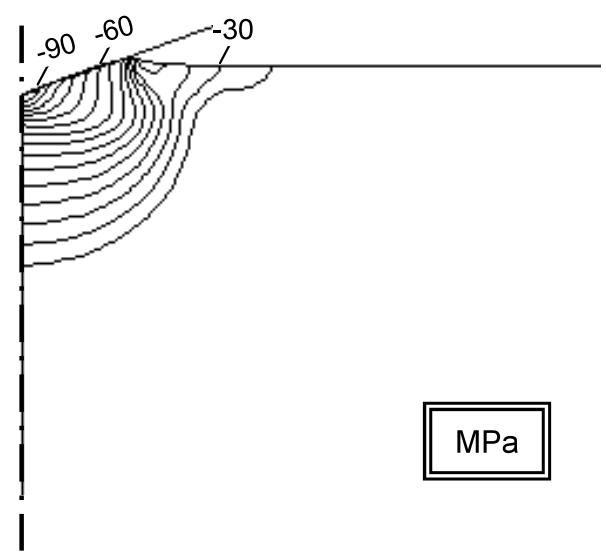

(b) Hydrostatic stress

Fig. 7 Contour maps of stress near contact area between indenter and surface rate due to power-law creep. After $1200 \mathrm{~s}$, there is no change in the stress distribution, but the level of stress decreases with self-similarity. Figure 8 (b) shows the evolution of hydrostatic stress. Not only equivalent stress but also hydrostatic stress decreases as the holding time increases, but a gradient of hydrostatic stress appears after $1200 \mathrm{~s}$. The hydrostatic stress is also higher than that the yield stress. Under multiaxial stresses, although there is a reduction in equivalent stress, residual hydrostatic stress appears. From Eqs. (3)-(5), atoms move from the indenter contact area and deformation due to diffusion takes place. The effective zone of the diffusion expands to over $50 \mu \mathrm{m}$ after $1200 \mathrm{~s}$. In the indentation creep test, the transition in the creep mechanism occurred after $1000 \mathrm{~s}$, and the results of FEA agree qualitatively with these of experiments. Therefore, as the strain rate due to power-law creep decreases, diffusion creep is dominant in the indentation creep test. The transition period depends strongly on the creep properties. As the creep exponent becomes higher, the relaxation proceeds rapidly and higher hydrostatic stress remains. On the other hand, when the creep exponent is low, the transition from power-law creep to diffusion creep occurs slowly.

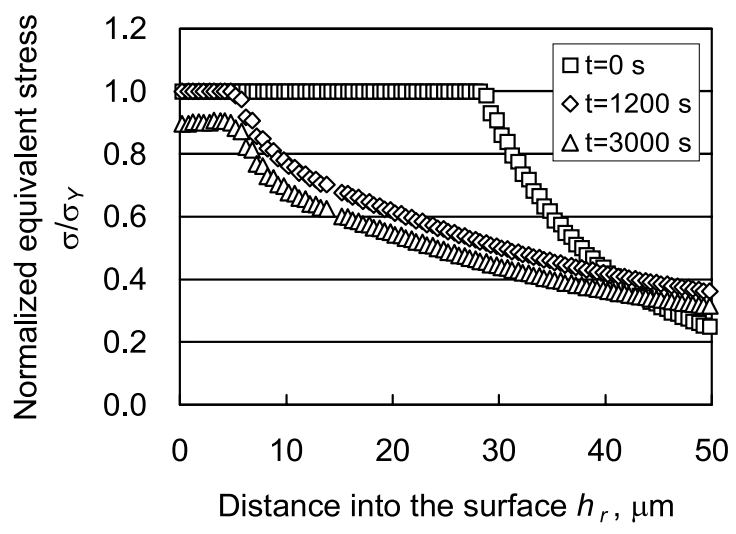

(a) Equivalent stress

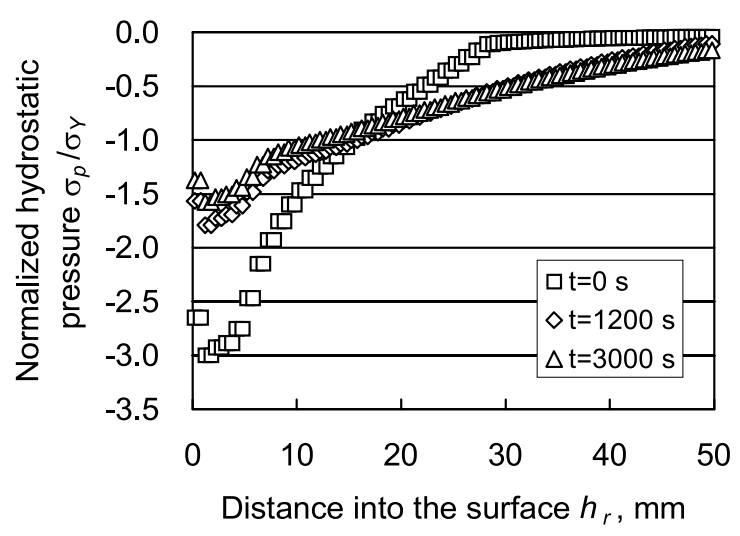

(b) Hydrostatic stress

Fig. 8 Evolution of stress in vicinity of tip of indenter 


\section{Conclusion}

The nanoindentation creep test for $\mathrm{Sn}-37 \mathrm{~Pb}$ was carried out, and the creep mechanism of low temperature melting point alloys was examined. The focus was on the effect of diffusion on creep behavior under multiaxial stresses. Results obtained are summarized as follows:

(1) The estimated creep exponent from the evolution of hardness in the nanoindentation creep test for $\mathrm{Sn}-37 \mathrm{~Pb}$, a low temperature melting point alloy, is 6 . It is higher than that of the bulk and close to that of single-crystal tin.

(2) As holding proceeds, the estimated stress sensitivity decreases to $n=1$, which is the creep exponent of diffusion creep.

(3) Plastic deformation due to the tip of the indenter is dominant in the early stages, and granular deformation is dominant after a decrease in the hardness. This implies that the mechanism of creep changes during nanoindentation creep.

( 4 ) FEA with an elastic-plastic-creep model reveals that the multiaxial stress field appears below the indenter. The equivalent stress is reduced on the basis of power-law creep, and residual hydrostatic stress becomes dominant.

(5) The transition from power-law creep due to equivalent stress to diffusion creep due to the gradient of hydrostatic stress occurs during nanoindentation creep.

\section{References}

(1) Ashby, M.F., A First Report on DeformationMechanism Maps, Acta Metall., Vol.20 (1972), pp.887-897.

( 2 ) Weertman, J., Discussion of: An Experimental Relation Defining the Stress Dependence of Minimum Creep Rate in Metals, Trans. Metal. Soc. A.I.M.E., Vol.227 (1963), pp.1475-1476.

( 3 ) Coble, R.I., A Model for Boundary Diffusion Controlled Creep in Polycrystalline Materials, J. Appl. Phys., Vol.34 (1963), pp.1679-1682.

( 4 ) Herring, C., Diffusional Viscosity of a Polycrystalline Solid, J. Appl. Phys., Vol.21 (1950), pp.437-445.

( 5 ) Harper, J. and Dorn, J.E., Viscous Creep of Aluminum near Its Melting Temperature, Acta Metall., Vol.5, No.11 (1957), pp.654-665.

( 6 ) Langdon, T.G., Creep at Low Stresses: An Evaluation of Diffusion Creep and Harper-Dorn Creep as Viable Creep Mechanisms, Metall. Mater. Trans. A-Phys. Metall. Mater. Sci., Vol.33 (2002), pp.249-259.

( 7 ) Mayo, M.J. and Nix, W.D., A Micro-Indentation Study of Superplasticity in $\mathrm{Pb}, \mathrm{Sn}$, and $\mathrm{Sn}-38 \mathrm{wt} \% \mathrm{~Pb}$, Acta Metal., Vol.36, No.8 (1988), pp.2183-2192.

( 8 ) Sargent, P.M. and Ashby, M.F., Indentation Creep, Mater. Sci. Technol., Vol.8, No.7 (1992), pp.594-601.

(9) Fujiwara, M. and Ohtsuka, M., Characterization of Micro-Indentation Creep in $\beta$-Sn Single Crystals at Elevated Temperatures, J. Japan Inst. Metals, (in Japanese), Vol.63, No.6 (1999), pp.760-769.

(10) Zhang, K. and Weertman, J.R., The Influence of Time, Temperature, and Grain Size on Indentation Creep in High-Purity Nanocrystalline and Ultrafine Grain Copper, Appl. Phys. Lett., Vol.85, No.22 (2004), pp.51975198.

(11) Ogawa, T., Miyamoto, A., Ohshimizu, K. and Ohsawa, T., Evaluation of Mechanical Properties of $\mathrm{Sn}-\mathrm{Pb}$ and Sn-Ag Eutectic Solders Using Hardness Testing, Journal of the Society of Materials Science, Japan, (in Japanese), Vol.49, No.6 (2000), pp.666-671.

(12) Chu, S.N.G. and Li, J.C.M., Impression Creep; A New Creep Test, J. Mater. Sci., Vol.12 (1977), pp.22002208.

(13) Needleman, A. and Rice, J.R., Plastic Creep Flow Effects in the Diffusive Cavitation of Grain Boundaries, Acta Metall., Vol.28 (1980), pp.1315-1332.

(14) Shibutani, T., Kitamura, T. and Ohtani, R., Creep Cavity Growth under Interaction between Lattice Diffusion and Grain Boundary Diffusion, Metall. Mater. Trans. A-Phys. Metall. Mater. Sci., Vol.29 (1998), pp.25332542.

(15) Chang, T.-J., Kagawa, K.I., Rice, J.R. and Sills, L.B., Non-Equilibrium Models for Diffusive Cavitation of Grain Interfaces, Acta Metall., Vol.27 (1979), pp.265284.

(16) Kitamura, T., Ohtani, R., Yamanaka, T. and Hattori, Y., Cavity Growth in Polycrystalline Materials under Grain Boundary Diffusion Creep and Transition from Cavity to Crack, JSME Int. J., Ser.A, Vol.38, No.4 (1995), pp.581-587.

(17) Shibutani, T., Kitamura, T. and Ohtani, R., Effect of Grain Boundary/Interface Network on Fracture due to Atom Migration Induced by Stress in an LSI Conductor, Trans. Jpn. Soc. Mech. Eng., (in Japanese), Vol.65, No.640, A (1999), pp.2497-2503.

(18) Cocks, A.C.F., A Finite Element Description of GrainBoundary Diffusion Processes in Ceramic Materials, Applied Solid Mechanics, Vol.3 (1989), pp.30-42.

(19) Mukai, M., Kawakami, T., Takahashi, K., Kishimoto, K. and Shibuya, T., Thermal Fatigue Life of Solder Bumps in BGA, JSME Int. J., Ser.A, Vol.41, No.2 (1998), pp.260-266. 